\title{
Understand how and why Glutathione is Important?
}

\section{Opinion}

Glutathione is an incredibly important compound, that the body makes in every cell, and is involved in a wide variety of functions. Most people who know of glutathione, think of it in terms of an anti-oxidant or part of the cellular redox mechanism. And it is. In fact, it is the most important anti-oxidant there is for a variety of reasons:

a. It deals with a variety of free radicals, whereas most antioxidants will only deal with one kind of free radical.

b. It operates everywhere: in the cell; the cell membrane; outside of the cell. Whereas most anti-oxidants operate in only one location.

c. It re-stabilizes itself whereas most anti-oxidants turn into free radicals once they have given away an electron.

d. It also re-stabilizes other anti-oxidants so that they can be useful again.

Consequently, glutathione is the most important anti-oxidant there is. Some researchers claim that it is 1000 s of times more important than any other anti-oxidant. But glutathione is not only known for its anti-oxidant capacity, it is also hugely involved in the detoxification processes. The major detoxification organ is the liver, of course, but glutathione operates as an anti-oxidant in every cell in the body. It does have its highest concentration in the liver (second highest concentration is in the eyes) and for a very good reason. There are a number of different detoxification pathways in the liver, but whether we look at the two-stage detox or the three stages or any other detoxification pathway; you usually find glutathione at the last step.

Glutathione is involved in the regulation of virtually all hormones, whether directly or indirectly through other compounds like nitric oxide, which of course, glutathione regulates directly. Nitric oxide is subsequently important for vasodilation, the immune system and regulating various hormones.

Glutathione is also recognized as the one compound that protects telomeres, which is often referred to as the aging marker. While many different compounds, usually anti-oxidants, protect telomeres, glutathione is the only compound that is known to provoke the growth of new telomeres and is therefor primary when it comes to anti-aging processes.

In addition, to protecting the telomeres, glutathione is also an important component of DNA protection. It not only protects DNA from going sideways, but also corrects or eliminates ones that do go sideways, therefore being a huge component of the anti-cancer process in the body.

Glutathione is also responsible for several different types of cellular transport, usually with amino acids. Red blood cells cannot uptake or release either oxygen or C02 without sufficient glutathione and are therefore hugely important to the respiratory/cardio system and maintaining healthy $\mathrm{pH}$ in the

Opinion
Volume 7 Issue 6 - 2017
Holly Fourchall**
Choices unlimited for Health \& Wellness Ltd, Canada
*Corresponding author: Holly Fourchalk, Choices Unlimited
for health and wellness, 14751 34a Ave, Surrey, BC V4P 0B3,
Canada, Tel: +1 604-764-5203,
Email: holly@choicesunlimited.ca
Received: January 23, 2017 | Published: July 07, 2017

blood. Most immune cells cannot develop or function effectively without sufficient glutathione. In addition to which, glutathione is also involved in the regulating the balance between various immune compounds like T1 \& T2.

However, even though glutathione can be found in various foods and is certainly sold as a nutraceutical, they are not of much help. The three amino acid compound usually breaks down in the hydrochloric acid of the stomach and then we lose most the unstable amino acid, cysteine, which is why $\mathrm{N}$-acetyl-cysteine was created. On the other hand, even if it didn't break down, it is too large to transport or diffuse into a cell.

Consequently, glutathione needs to be synthesized and regulated in each cell. Another way of administering glutathione is in the nano form. But I always question the validity of any nano sized particle. How is it that a molecule is the $1 / 5000$ th its normal size is going to interact with enzymes of receptors that are now massive in comparison. Nano particles are also used to increase bioavailability, but if the compound doesn't go through it's natural receptor processes, how are the required subsequent processes going to get triggered so that the cell can utilize the nutrient?

Another delivery system involves injecting glutathione directly into the blood stream- which may be advantageous if the client has a serious blood toxic. But there needs to be caution, because the body's natural feedback systems, then tell the cells to stop producing glutathione as there is an overload.

So again, we are back to the cells regulating and synthesizing glutathione. How do we make sure that the cells can in fact regulate and synthesize glutathione? By making sure that the methylation cycles (methionine, folate, biopterin and uric) are working effectively; that there are sufficient mitochondria in the different types of cells; that the mitochondria can produce sufficient ATP for the cell; and by making sure that all the necessary genes are in fact, "turned on". Which of course, is all dependent on a healthy diet and gut that can digest and absorb the required nutrients for all the different functions; a healthy blood stream with sufficient transport mechanisms to and from the liver; and a healthy liver to create the necessary compounds that the blood delivers. 
Once again, we need to able to understand how and why cells function as they do, so that we can support the body's natural wisdom and capacity to regulate and synthesize glutathione, which is required for so many different healthy functions. 